\title{
AMYLOSE/AMYLOPECTIN SIMPLE DETERMINATION IN ACID HYDROLYZED TAPIOCA STARCH
}

\author{
KARNTARAT WUTTISELA ${ }^{1}$, SUJIN SHOBSNGOB ${ }^{2}$, WANNAPONG TRIAMPO ${ }^{1,3,4}$, \\ DARAPOND TRIAMPO ${ }^{1,2 * 3}$
}

\author{
${ }^{1}$ Institute for Innovation and Development of Learning Process, Mahidol University, \\ Rama 6 Rd., Rajchataywee, Bangkok 10400, Thailand \\ ${ }^{2}$ Department of Chemistry (R3/1), Faculty of Science (Salaya Campus), Mahidol University, \\ Phuttamonthon Sai 4 Rd., Salaya, Nakhon Pathom 73170, Thailand \\ ${ }^{3}$ Center of Excellence for Vectors and Vector-Borne Diseases, Faculty of Science, \\ Mahidol University, Salaya Campus, Phuttamonthon Sai 4 Rd., Nakhon Pathom 73170, Thailand \\ ${ }^{4}$ Department of Physics (BIOPHYSICS), Faculty of Science, Mahidol University, \\ Rama 6 Rd, Rajchatavee, Bangkok 10400, Thailand \\ (Received: May 28, 2007 - Accepted: May 2, 2008)
}

\begin{abstract}
Analysis of the shift of wavelength maximum using a rapid colorimetric method was used to determine the ratio of amylose:amylopectin (Am:Ap) in acidhydrolyzed tapioca starch. The absorbance maximum of $600 \mathrm{~nm}$ (Am:Ap of tapioca starch $\approx 22: 78)$ moved to shorter wavelengths $(590,585,570,560$, and 534 $\mathrm{nm})$ as the decrease of the Am:Ap ratio due to hydrolysis of shorter chains that are not be able to form a complex with iodine. The amount of amylopectin itself may be unaltered or slightly decreased but the decrease in amylose caused a decrease in Am:Ap ratio.
\end{abstract}

Keywords: amylose, amylopectin, acid hydrolysis, colorimetric

\section{INTRODUCTION}

Starch is the major carbohydrate reserve of plant tubers and seed endosperm. ${ }^{1}$ The largest source of starch is maize, wheat, potato, tapioca, and rice. Starch is widely used as thickener, water binder, emulsion stabilizer, and gelling agent. Each starch granule typically contains amylopectin, a linear chain of $(1 \rightarrow 4)$ - $\alpha$-D-glucose residues connected through branched $(1 \rightarrow 6)-\alpha$ linkages, and a much larger number of the smaller amylose, $\alpha(1 \rightarrow 4)$ linearly linked D-glucopyranosyl residues.

Amylose is a hydrocolloid. Its extended conformation causes the high viscosity of water-soluble starch which varies relatively little with temperature. The extended loosely helical chains possess a relatively hydrophobic inner surface that is not able to hold water. Amylose forms useful gels and films. Its association and crystallization (retrogradation) on cooling and storage decrease storage stability, causing shrinkage and the release of water (syneresis). Increasing amylose concentration decreases gel stickiness, but increases gel firmness. Amylopectin interferes with the interaction between amylose chains (and retrogradation) and its solution can lead to an initial loss in viscosity and followed by a more slimy consistency.

The simplest and most common starch modification is by acid hydrolysis, which is widely used in food, paper, textile, and pharmaceutical industries. ${ }^{2,3}$ This is conducted mainly by soaking the starch in dilute acid. ${ }^{4}$

In normal starch, the amylose:amylopectin (Am:Ap) ratio is $\approx 1: 4$. Meals made with high amylose rice ${ }^{5}$ or cookies prepared from high amylose starches $^{6}$ induce a lower postprandial glucose response in the blood. In addition, consumption of high amylose diet for several weeks results in lower postprandial concentration of insulin and lower fasting concentration of triacylglycerol and cholesterol. ${ }^{7}$ Starch films made from different Am:Ap ratio have different properties. Amylose in starch gives stronger film whereas amylopectin generally leads to lower mechanical properties. ${ }^{8,9}$ Physical and chemical properties of starch films can be tailored by adjusting Am:Ap ratio. ${ }^{10,11}$

The present work describes the use of a simple colorimetric method to analyze Am:Ap ratio via shift of wavelength maximum $\left(\lambda_{\max }\right)$ seen at different stages of acid hydrolysis of tapioca starch.

\section{EXPERIMENTAL}

\section{Materials}

Tapioca starch was obtained from a commercial source in Thailand. Hydrochloric acid and sodium hydroxide were purchased from Merck KG, Darmstadt, Germany. Potassium iodide, iodine, acetic acid, pure potato amylose, and ethanol were purchased from Sigma Co., Ltd., USA.

\section{Acid hydrolysis of tapioca starch}

Starch was suspended at $10 \%(\mathrm{w} / \mathrm{v})$ in $0.7 \mathrm{M}$ aqueous $\mathrm{HCl}$ and $2.0 \mathrm{M}$ aqueous $\mathrm{HCl}$ for $0.5 \mathrm{~h}, 1.5 \mathrm{~h}, 2.5 \mathrm{~h}$, and $3.5 \mathrm{~h}$ at $50^{\circ} \mathrm{C}$ with constant stirring and then the suspensions were neutralized with $\mathrm{NaOH}$ to a $\mathrm{pH}$ of $7.0 \pm 0.5$. The acid-modified starch was recovered by centrifugation and freeze-dried.

\section{Preparation for colorimetric absorption analysis}

A fraction $(0.10 \mathrm{~g})$ of the acid-modified starch was dissolved in a mixture of $1 \mathrm{ml}$ of $95 \%$ ethanol and $9 \mathrm{ml}$ of $2 \mathrm{M} \mathrm{NaOH}$ at $95^{\circ} \mathrm{C}$. When the starch was dissolved completely, this solution was diluted with deionized water in a 100 $\mathrm{ml}$ volumetric flask. An aliquot of $5.0 \mathrm{ml}$ was mixed with $2.0 \mathrm{ml}$ of $1 \mathrm{M}$ acetic acid, $2.0 \mathrm{ml}$ of freshly prepared iodine reagent and $91.0 \mathrm{ml}$ of deionized water. The iodine reagent was prepared by dissolving $0.20 \mathrm{~g}$ of iodine and $2.0 \mathrm{~g}$ of potassium iodide with deionized water in a $100 \mathrm{ml}$ volumetric flask.

\section{Colorimetric absorbance analysis}

Absorbance spectra from 400 to $800 \mathrm{~nm}$ were recorded for all samples with a $1 \mathrm{~cm}$ path-length cuvette in a Cary 300Bio UV/Visible spectrophotometer.

A standard curve was plotted for various concentrations of pure potato amylose. Potato starch was used as the standard because potato and tapioca starch have approximately the same amylose and amylopectin content. ${ }^{1,12}$ Spectra for blanks of the amylose solutions and the iodine reagent were also recorded.

Figure 1 shows a typical absorbance spectrum. Data analysis of the spectra was conducted by smoothing of the spectra. Two separate pieces of data were then extracted: the absorbance at $610 \mathrm{~nm}\left(\lambda_{610}\right.$, (a) in Figure 1), which was used to determine the Am:Ap ratio, and the wavelength with maximum absorbance $\left(\lambda_{\max }\right)$ within a given range of $400-800 \mathrm{~nm}$ obtained using a mathematical algorithm (b in Figure 1). ${ }^{13}$ 


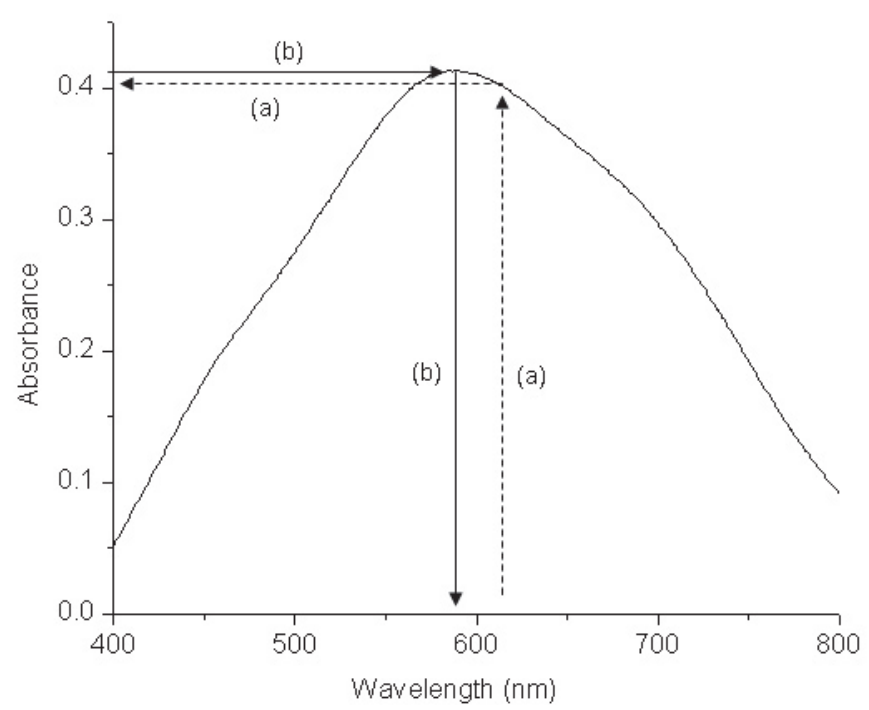

Figure 1. Typical absorbance spectrum of native starch (a) Absorbance at $610 \mathrm{~nm}$ used to determine the amylose content (percent amylose). (b) Maximum absorbance $\left(\lambda_{\max }\right)$ determined using a mathematical algorithm.

\section{RESULTS AND DISCUSSION}

The Am:Ap ratio in native tapioca starch was approximately 22:78. Figure 2 shows that the Am:Ap ratio obtained by an iodometry method at a single wavelength $\left(\lambda_{610}\right)$ measurement decreased with hydrolysis time using $2 \mathrm{M} \mathrm{HCl}$, but not with $0.7 \mathrm{M} \mathrm{HCl}$. This change was seen after 30 min of incubation.

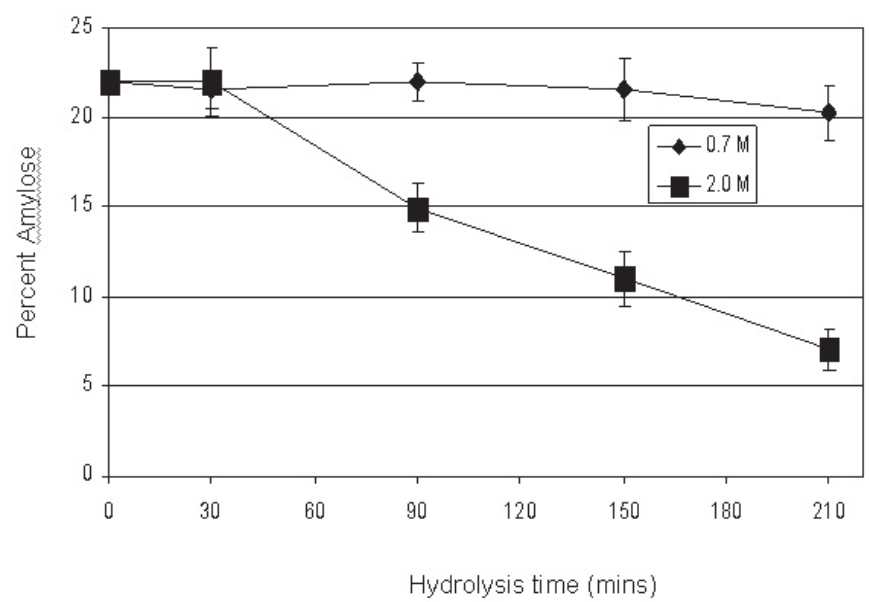

Figure 2. Plot of percent amylose content obtained from a single wavelength $\left(\lambda_{610}\right)$ iodometry measurement with $\mathrm{HCl}$ hydrolysis time $(\diamond) 0.7 \mathrm{M}$ $\mathrm{HCl}$, and $(\square) 2.0 \mathrm{M} \mathrm{HCl}$.

There was a shift of $\lambda$ to shorter wavelengths with hydrolysis time (Figure 3). The change was more pronounced using $2 \mathrm{M} \mathrm{HCl}$. A good correlation was obtained between $\lambda_{\text {max }}$ and amylose content for percent amylose content between $10-20$ (Figure 4).

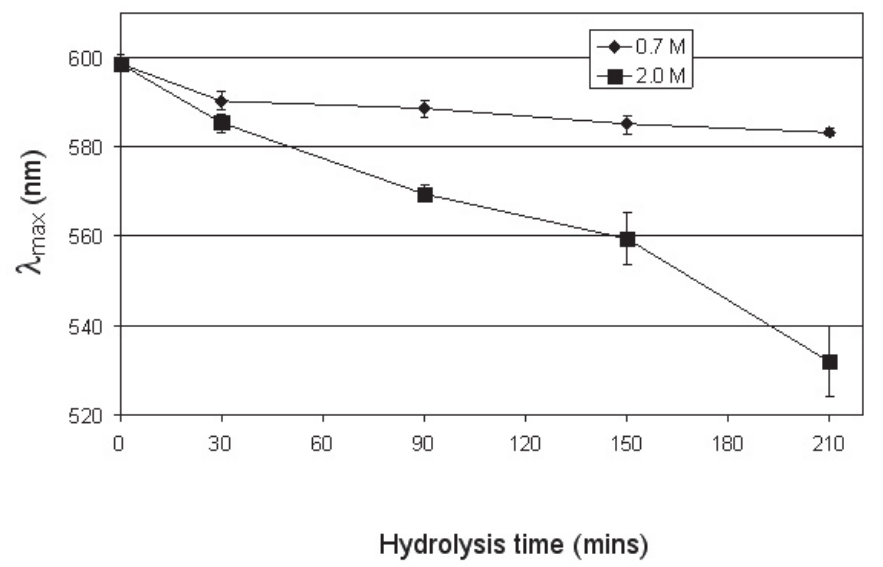

Figure 3. Plot of absorbance maximum $\left(\lambda_{\max }\right)$ determined mathematically with $\mathrm{HCl}$ hydrolysis time $(\diamond) 0.7 \mathrm{M} \mathrm{HCl}$, and $(\square) 2.0 \mathrm{M} \mathrm{HCl}$.

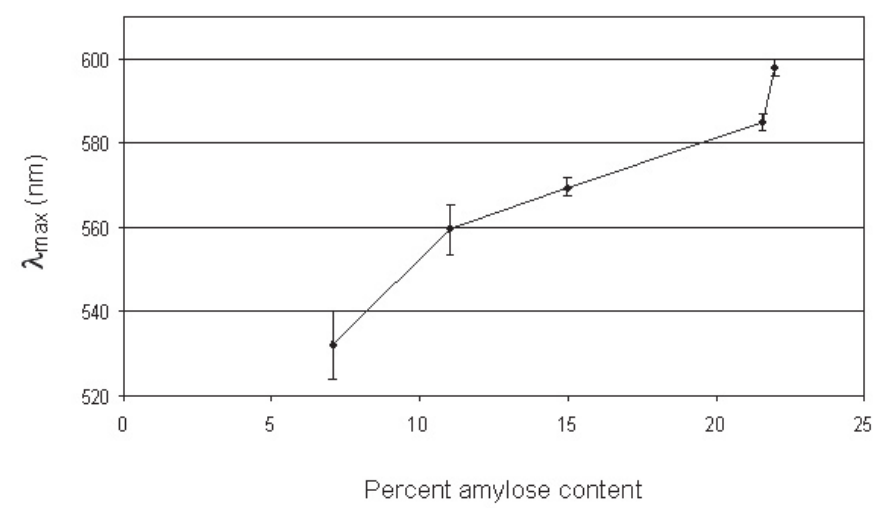

Figure 4. Plot of $\lambda_{\max }$ and percent amylose content.

Starch is hydrolyzed by acid into shorter glucose chains. With the iodometry method, approximately 6 glucose units are required to form a complex with iodine that will give a measurable absorption at $610 \mathrm{~nm} .{ }^{14}$ With $0.7 \mathrm{M} \mathrm{HCl}$, hydrolysis does not change the Am:Ap ratio because there is competition between hydrolysis of the amorphous part of the amylopectin into shorter glucose chains capable of forming a complex with iodine resulting in an increase in the Am:Ap ratio and hydrolysis of the original amylose into shorter chains that are not able to form a complex with iodine (resulting in decrease in the Am:Ap ratio). With $2.0 \mathrm{M} \mathrm{HCl}$, this effect was observed during the first $30 \mathrm{~min}$ of hydrolysis. From $30-210 \mathrm{~min}$ of hydrolysis by $2.0 \mathrm{M}$ $\mathrm{HCl}$, the observation of competition between hydrolysis of the amorphous part of amylopectin and hydrolysis of the original amylose is no longer reflected in the analysis, perhaps as all the amorphous part of amylopectin has been hydrolyzed. What was observed was the decrease of the Am:Ap ratio due to hydrolysis of shorter chains that are not be able to form a complex with iodine. The amount of amylopectin itself may be unaltered or slightly decreased but the decrease in amylose caused decrease in Am:Ap ratio.

\section{CONCLUSION}

In summary, it has been demonstrated that $\lambda_{\max }$ shifts to shorter wavelength with decreasing Am:Ap ratio, and provides a simple method of determining Am:Ap ratio following acid hydrolysis of tapioca starch.

\section{ACKNOWLEDGEMENTS}

This work has been supported in part by Postgraduate Education and Research Program in Chemistry (PERCH), The Thai Center of Excellence for Physics (Integrated Physics Cluster), Coordinating Center for Thai Government Science and Technology Scholarship Students of the National Science and Technology Development Agency (CSTS, NSTDA), Thailand Research 
Funding Agency (TRF), and Thailand National Center for Engineering and Biotechnology (BIOTEC).

\section{REFERENCES}

1. A. Buleon, P. Colonna, V. Planchot, S. Ball, Int. J. Biol. Macromol. 23, 85 (1998).

2. Y.J. Wang, V.D. Truong, L. Wang, Carbohydr. Polym. 52, 327 (2003).

3. H. Puchongkavarin, W. Bergthaller, S. Shobsngob, S. Varavinit, Starch/ Stärke 55, 464 (2003).

4. R. Hoover, Food Rev. Int. 16, 369 (2000).

5. M.S. Goddard, 0. Young, R. Marcus, Am. J. Clin. Nutr. 39, 388 (1984).

6. K.M. Behall, D.J. Scholfield, J. Canary, Am. J. Clin. Nutr. 47, 428 (1988).
7. K.M. Behall, D.J. Scholfield, I. Yuhaniak, J. Canary, Am. J. Clin. Nutr. 49, 337 (1989).

8. R.N. Tharanathan, Trends in Food Science and Technology 14(3), 71 (2003).

9. S. Mali, M.V.E. Grossmann, M.A. Garcia, M.N. Martino, N.E. Zaritzky, $J$. Food Engineering 75(4), 453 (2006).

10. H.D. Belitz, W. Grosh, Food Chemistry, Springer, Heidelberg, 1999.

11. J. Delvile, C. Joly, P. Dole, C. Bliard, Carbohydr. Polym. 49, 71 (2002).

12. A.M. Myers, M.K. Morell, M.G. James, S.G. Ball, Plant Physiol. 122, 989 (2000).

13. S.J. McGrance, H.J. Cornell, C.J. Rix, Starch/Stärke 50, 158 (1998).

14. R.E. Rundle, J. Am. Chem. Soc. 65, 544 (1943). 